\title{
The pathology of the eye in armadillos experimentally infected with Mycobacterium leprae
}

\author{
F BRANDT, * H M ZHOU, $\dagger \mathrm{Z}$ R SHI, $\dagger$ \\ J KADZDA, $\ddagger$ A M DHOPLE, $\S$ A KOLK $q$ \\ D S SCHMIDT** \\ * Ludwigstrasse 97, 8265 Neuötting, Federal Republic of \\ Germany; $\dagger$ Departments of Pathology and Ophthalmology, \\ Qingdao College, China; $\ddagger$ Forschungsinstitut Borstel, Borstel, \\ Federal Republic of Germany; $\S$ Medical Research Institute \\ Florida, Institute of Technology, Melbourne, Florida, USA; \\ I Royal Tropical Institute, Amsterdam, The Netherlands; and \\ Pathologisches Institut Traunstein, Federal Republic of \\ Germany**
}

\section{Accepted for publication 27 October 1989}

Summary One hundred and twenty-seven eyes from 66 Mycobacterium leprae inoculated armadillos were studied histologically and some ultrastructurally.

Inflammatory reactions were found in the following extraocular tissues: the eyelid, including the orbicularis muscle and the third eyelid, extraocular muscles, tear gland and Harder's gland.

The early and slight changes of the intraocular tissues, small amounts of lymphocytes, plasma cells and macrophage infiltrations were confined to the area around the anterior angle specifically within the trabeculae and the adjacent ciliary body, the root of the iris and the limbus region of the cornea.

But in the cases with severe lesions the whole uvea was densely infiltrated with large, foamy macrophages intermingled with small amounts of lymphocytes, plasma cells and frequently, neutrophils. No specific necrosis of the granulomas was seen. No explanation for the neutrophil infiltrations was given.

The lesions in the cornea were significantly less severe than those in the uvea. Retinal lesions comprised of macrophage infiltrations were all obvious extensions of the adjacent uvea lesions. Acid-fast bacilla (AFB) were found within all tissues. The infection of the intraocular tissues in the armadillo eyes seemed to be mainly, if not solely, haematogenous.

\section{Introduction}

Although the incidence of leprosy is declining in many parts of the world the prevalence of this disease is still considerable in many countries. The eyes of leprosy patients are 
damaged either by the disease itself or because of lagophthalmos and insensitivity of the eyes.

The clinical aspects of eye diseases in leprosy have been well documented, but the pathological studies of the eye in human patients are few, ${ }^{1}$ this is due to the fact that eye specimens of patients are hard to get. Whenever eyeballs were available, they were almost always at the end stage of disease and had been treated over a long period of time. What we know about the pathology of the whole human eyeball in leprosy is based on limited observations of human specimens. In order to gain an overview of the pathology of the eye in leprosy, and to know the pathogenesis of it animal model studies are necessary.

Since the discovery that armadillos could be artificially infected with Mycobacterium leprae, ${ }^{2,3}$ these animals have been used both as a source of the causative organism for other research and as an animal model of human leprosy in different fields of research. ${ }^{4}$ In order to know more about the pathology of the eye lesions in this animal model, 130 eyes from 68 experimentally infected armadillos were studied histologically and in several cases ultrastructurally.

\section{Material and methods}

One hundred and thirty eyes were studied from $68 \mathrm{M}$. leprae infected armadillos (Dasypus novemcinctus). The eyes came from the following research institutes: Forschungsinstitut Borstel of West Germany, Royal Tropical Institute of Amsterdam, Holland and Florida Institute of Technology, Melbourne, Florida, USA. Six of these animals had only one eye specimen available.

The animals were inoculated intravenously with $M$. leprae through the femoral vein and with a dosage of $10^{8}$ bacilli in $1 \mathrm{ml}$ of saline. Between 5 and 60 months after inoculation the animals were put to sleep and the eyes removed, 59 of them with the eyelids and intraorbital tissues intact. The specimens were fixed in buffered formaldehyde or glutaraldehyde. Paraffin sections of these specimens were made and stained either with haematoxylin-eosin, with modified Fite-Faraco (FF) method for acid-fast bacilli (AFB) after depigmentation with potassium permanganate, ${ }^{5}$ with Gomori or with PAS methods. The eys of 3 armadillos fixed in glutaraldehyde were studied ultrastructurally under a Philip electron microscope, after having been embedded in Epon and cut with an LKB microtome.

We categorized the lesions of the eyes of these animals into four groups according to the severity of the reactions: the group with minimal lesions $(+-)$ included the eyes with very small amounts of lymphocytes and/or plasma cell infiltrations, usually around the anterior angle, in the ciliary body and occasionally in the iris and choroid. If there were small numbers of macrophages present, the cytoplasm in the macrophages was not very abundant. The one plus group $(+)$ included the eyes with lymphocytes plasma cell and large macrophage (with extensive cytoplasm) infiltrations around the anterior angle and in the iris, ciliary body and choroid. The two plus $(++)$ group included the cases with significant infiltrations of plump macrophages in the ciliary body, iris and choroid, with slight to moderate thickening of these structures. The three plus $(+++)$ group included the cases with the whole uvea tract densely infiltrated with plump macrophages and the uvea significantly thickened. 


\section{Results}

Of the 130 eye specimens from the inoculated armadillos, one eye was excluded from the study because of its autolytic changes. Both eyes from another animal were also excluded because there was acute keratitis in both eyes with neutrophile infiltrations in the whole cornea stroma and no AFB or other significant lesions in other ocular tissues.

In the remaining 127 eyes, the following changes in various tissues were found, see Tables 1 and 2 .

\section{EYELID}

In 59 of the 127 eye specimens, eyelids were present. In the skin of 56 of these eyelids $(95 \%)$, granulomatous lesions were found. There were gatherings of large macrophages and foam cells in the dermis, usually intermingled with small numbers of lymphocytes, plasma cells and neutrophiles (Figure 1). Where the infiltrations were not very dense, the lesions were more prominent around the adnexa of the skin and around the nerves and blood vessels. The perineurium of some nerves was thickened with perineurial cell proliferation. In 21 of the 52 eyes $(40 \%)$ in which the orbicularis muscles were visible, the muscles were infiltrated with macrophages or foam cells and other inflammatory cells

Table 1. Number of eyes with lesions in various ocular tissues

\begin{tabular}{|c|c|c|c|c|c|}
\hline & \multicolumn{4}{|c|}{ Lesion group } & \multirow[b]{2}{*}{ Total } \\
\hline & +- & + & ++ & +++ & \\
\hline $\begin{array}{l}\text { Number of eyes } \\
\text { in this group }\end{array}$ & 42 & 52 & 21 & 12 & 127 \\
\hline \multicolumn{6}{|l|}{$\begin{array}{l}\text { No. of eyes } \\
\text { with lesions } \\
\text { found in the } \\
\text { tissue of: }\end{array}$} \\
\hline Cornea & $4(10 \%)^{*}$ & $7(13 \%)$ & $10(48 \%)$ & $9(75 \%)$ & $30(24 \%)$ \\
\hline Sclera & $1(2 \%)$ & $3(6 \%)$ & $5(4 \%)$ & $9(75 \%)$ & $18(14 \%)$ \\
\hline Limbus & $20(48 \%)$ & $40(77 \%)$ & $18(86 \%)$ & $11(92 \%)$ & $89(70 \%)$ \\
\hline Trabeculae & $37(88 \%)$ & $52(100 \%)$ & $21(100 \%)$ & $12(100 \%)$ & $122(96 \%)$ \\
\hline Iris & $6(14 \%)$ & $32(62 \%)$ & $21(100 \%)$ & $12(100 \%)$ & $71(56 \%)$ \\
\hline Ciliary body & $35(83 \%)$ & $52(100 \%)$ & $21(100 \%)$ & $12(100 \%)$ & $120(94 \%)$ \\
\hline \multicolumn{6}{|l|}{ Choroid } \\
\hline Ant. & $9(21 \%)$ & $45(87 \%)$ & $21(100 \%)$ & $12(100 \%)$ & $87(69 \%)$ \\
\hline Mid. & $6(14 \%)$ & $30(58 \%)$ & $17(81 \%)$ & $12(100 \%)$ & $65(51 \%)$ \\
\hline Post. & $6(14 \%)$ & $23(44 \%)$ & $13(62 \%)$ & $11(92 \%)$ & $53(42 \%)$ \\
\hline Retina & $0 / 42(0 \%) \dagger$ & $0 / 52(0 \%)$ & $2 / 19(11 \%)$ & $6 / 12(50 \%)$ & $8 / 125(6 \%)$ \\
\hline Optic nerve & $0 / 19(0 \%)$ & $0 / 22(0 \%)$ & $0 / 7(0 \%)$ & $0 / 5(0 \%)$ & $0 / 53(0 \%)$ \\
\hline Lens & $8 / 28(29 \%)$ & $14 / 45(31 \%)$ & $12 / 18(67 \%)$ & $6 / 11(55 \%)$ & $40 / 102(39 \%)$ \\
\hline
\end{tabular}

* The figure represents the number of the eyes in which lesions were found in that tissue. The figure in parenthesis represents the percentage of the eyes in which lesions in that tissue were found.

† The numerator represents the number of eyes in which AFB were found. The denominator represents the number of the eyes in which that tissue was distinct and AFB had been looked for. The figure in the parenthesis represents the percentage of the AFB positive cases. 
(Figure 2). Different quantities of AFB were found both within these infiltrated cells and outside them.

\section{CONJUNCTIVA}

Infiltrations of macrophages with foamy cytoplasms and AFB were found both in the bulbar and lid conjunctiva in 84 eyes (66\%) (Figure 3 ).

In the conjunctiva of the eyelid and fornix, small nodules of lymphocyte gatherings were observed. Scattered lymphocytes, plasma cells and Russell's bodies had infiltrated the conjunctiva elsewhere. In one case hair follicles with sebaceous glands were found in the palpebral conjunctiva facing the nictating membrane.

THE THIRD EYELID, NICTATING MEMBRANE

The nictating membrane was seen in 45 of the 127 eyes. In the connective tissues of 43 $(96 \%)$ of these third eyelids, large amounts of macrophages or foam cells, with many AFB in their cytoplasms were observed. Sometimes giant cells and neutrophiles and other cells were found (Figure 4). The cartilages of these eyelids appeared to be normal, but AFB were discovered both in the perichondrial cells and in the chondrocytes in 5 cases.

Table 2. Number of eyes and intraocular tissues in which AFB were found

\begin{tabular}{|c|c|c|c|c|c|}
\hline & \multicolumn{4}{|c|}{ Lesion group } & \multirow[b]{2}{*}{ Total } \\
\hline & +- & + & ++ & +++ & \\
\hline $\begin{array}{l}\text { No. of eyes } \\
\text { in this group }\end{array}$ & 42 & 52 & 21 & 12 & 127 \\
\hline $\begin{array}{l}\text { No. of eyes } \\
\text { with AFB looked for }\end{array}$ & 42 & 51 & 20 & 12 & 125 \\
\hline $\begin{array}{l}\text { No. of eyes } \\
\text { with AFB found }\end{array}$ & 13 & 46 & 20 & 12 & $91 / 125(73 \%)^{*}$ \\
\hline \multicolumn{6}{|l|}{$\begin{array}{l}\text { AFB found in } \\
\text { the tissues of: }\end{array}$} \\
\hline Cornea & $9 / 42(21 \%)^{*}$ & $25 / 50(50 \%)$ & $16 / 20(80 \%)$ & $10 / 12(83 \%)$ & $60 / 124(48 \%)$ \\
\hline Limbus & $11 / 42(17 \%)$ & $37 / 50(74 \%)$ & $16 / 20(80 \%)$ & $11 / 12(92 \%)$ & $75 / 124(60 \%)$ \\
\hline Trabeculae & $12 / 42(29 \%)$ & $36 / 49(73 \%)$ & $17 / 18(94 \%)$ & $11 / 11(100 \%)$ & $76 / 120(63 \%)$ \\
\hline Iris & $5 / 42(12 \%)$ & $28 / 49(57 \%)$ & $17 / 19(89 \%)$ & $11 / 11(100 \%)$ & $61 / 121(50 \%)$ \\
\hline Ciliary body & $13 / 42(31 \%)$ & $43 / 51(84 \%)$ & $19 / 20(95 \%)$ & $11 / 11(100 \%)$ & $86 / 124(69 \%)$ \\
\hline Choroid & $13 / 42(31 \%)$ & $46 / 51(90 \%)$ & $20 / 20(100 \%)$ & $12 / 12(100 \%)$ & $91 / 125(73 \%)$ \\
\hline Retina & $2 / 41(5 \%)$ & $9 / 50(18 \%)$ & $6 / 17(35 \%)$ & $10 / 11(91 \%)$ & $27 / 119(23 \%)$ \\
\hline Sclera & $7 / 42(17 \%)$ & $33 / 50(66 \%)$ & $19 / 20(95 \%)$ & $12 / 12(100 \%)$ & $71 / 124(57 \%)$ \\
\hline Optic nerve & $1 / 14(7 \%)$ & $2 / 16(13 \%)$ & $1 / 2 \quad(50 \%)$ & $2 / 6 \quad(33 \%)$ & $6 / 38 \quad(16 \%)$ \\
\hline Lens & $0 / 19(0 \%)$ & $1 / 37(2 \%)$ & $0 / 15(0 \%)$ & $3 / 9 \quad(33 \%)$ & $4 / 80 \quad(5 \%)$ \\
\hline Blood vessel & $3 / 42(7 \%)$ & $6 / 51(12 \%)$ & $4 / 20(20 \%)$ & $5 / 12(42 \%)$ & $18 / 125(14 \%)$ \\
\hline
\end{tabular}

* The numerator represents the number of eyes in which AFB were found. The denominator represents the number of the eyes in which that tissue was distinct and AFB were looked for. The figure in the parenthesis represents the percentage of the AFB positive tissues found. 


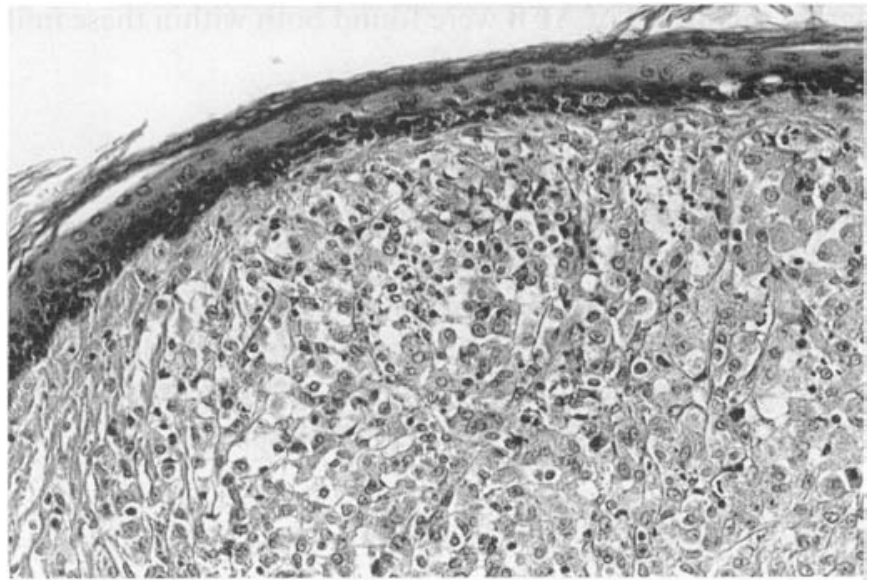

Figure 1. The cells infiltrating the dermis are mainly foam cells and smaller macrophages mixed with a small amount of other cells, including neutropheles. HE $284 \times$.

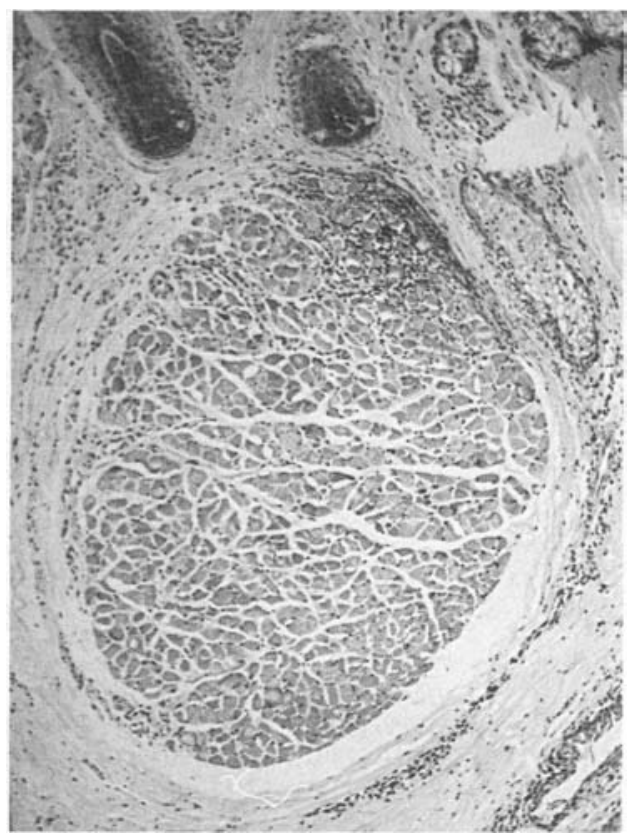

Figure 2. The obicularis muscle in the eyelid is infiltrated by inflammatory cells. HE $71 \times$.

\section{LACRYMAL GLAND}

Lacrymal and/or accessory lacrymal glands were found in 91 eyes. In 51 (56\%) of the 91 eyes lymphocyte infiltrations were found, some with macrophages and some with the gland partially destroyed (Figure 5). AFB were found in $43(55 \%)$ of the 78 glands studied. 


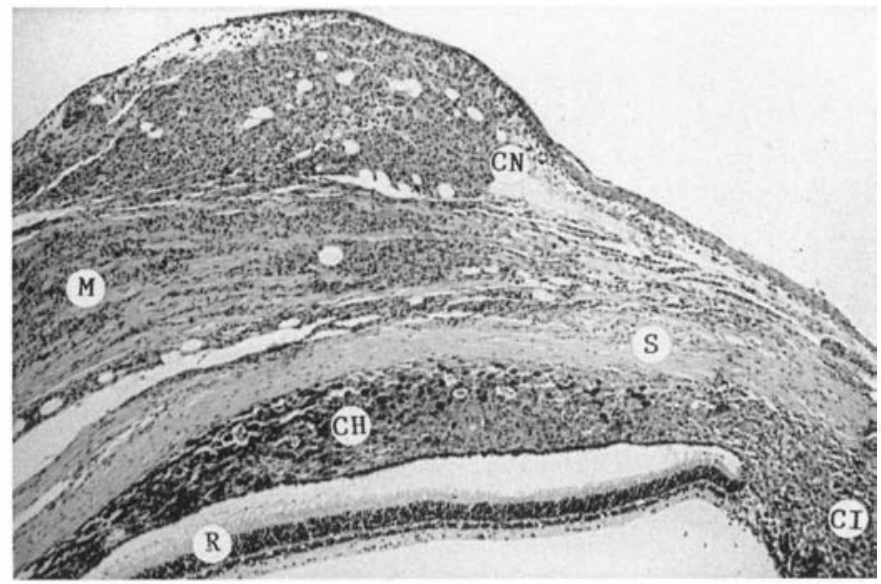

Figure 3. In the bulbar conjunctiva $(\mathrm{CN})$, a focus of macrophage infiltration is bulging outward. The infiltrations in the extraocular muscle $(\mathrm{M})$, choroid $(\mathrm{CH})$ and ciliary body $(\mathrm{CI})$ are also prominant. $\mathrm{R}$, retina; S, sclera. HE $71 \times$.

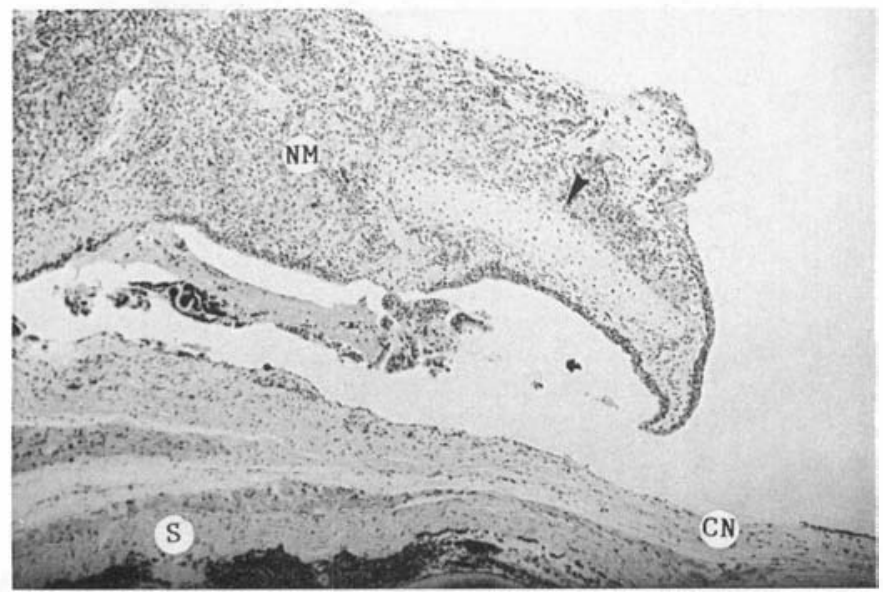

Figure 4. The stroma of the nictating membrane (NM) is heavily infiltrated with inflammatory cells, but the cartilage (arrow head) in the third eyelid seems intact. CN: conjunctiva, S: sclera. HE $71 \times$.

\section{EXTRAOCULAR MUSCLES}

Although 83 of the 127 muscles were intact, the muscles in 44 cases $(35 \%)$ were infiltrated with large macrophages or foam cells in great numbers (Figures 3 and 6). In some cases parts of the muscle had been replaced by there infiltrated cells and only a few muscle cells remained among a great number of foamy macrophages. Hypertrophy of the remaining muscle cells could be observed. A lot of AFB were to be seen in the infiltrating cells (Figure 7) in the muscle bundles. In the muscle cells of several animals, parasite sarcocystes were found filling the cytoplasm, usually with no inflammatory reaction involved. 


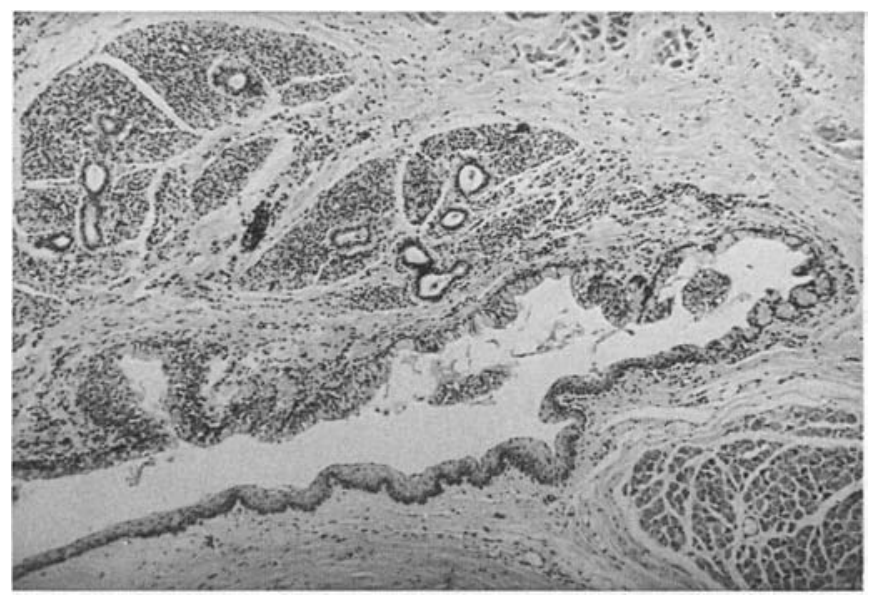

Figure 5. The accessory lacrimal gland within the palpebral conjunctiva is significantly infiltrated with macrophages. HE $71 \times$.

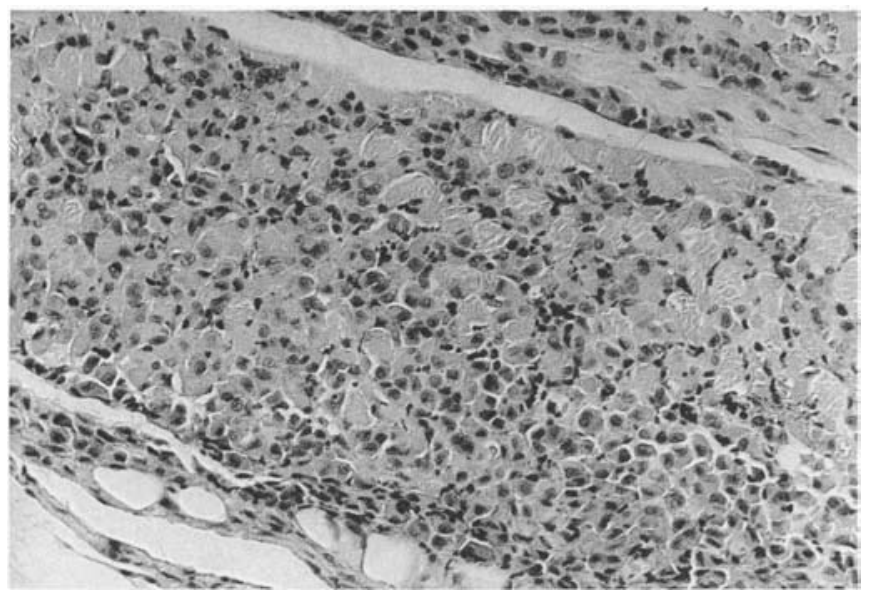

Figure 6. The extraocular muscle is infiltrated with large macrophages. Significant neutrophiles can be observed intermingling with these cells. HE $284 \times$.

\section{CORNEA}

The cell infiltration in the cornea was not extreme; usually only minimal cell infiltrations at limbus were found (Figures 8 and 9). The infiltrating cells were mostly comprised of some small or large macrophages, lymphocytes, plasma cells and sometimes neutrophiles. In some cases there were small round cells scattered around the small blood vessels, a normal constituent of the cornea of this species. In most of the cases, the epithelium and the endothelium of the cornea showed no changes, though in others some of these cells were swollen. In some specimens, sheets of endothelia were detached, leaving bulla-like spaces between them and the Descemet's membrane. AFB were found in the epithelium cells, in cells infiltrated between the lamellae of the stroma cells and within the 


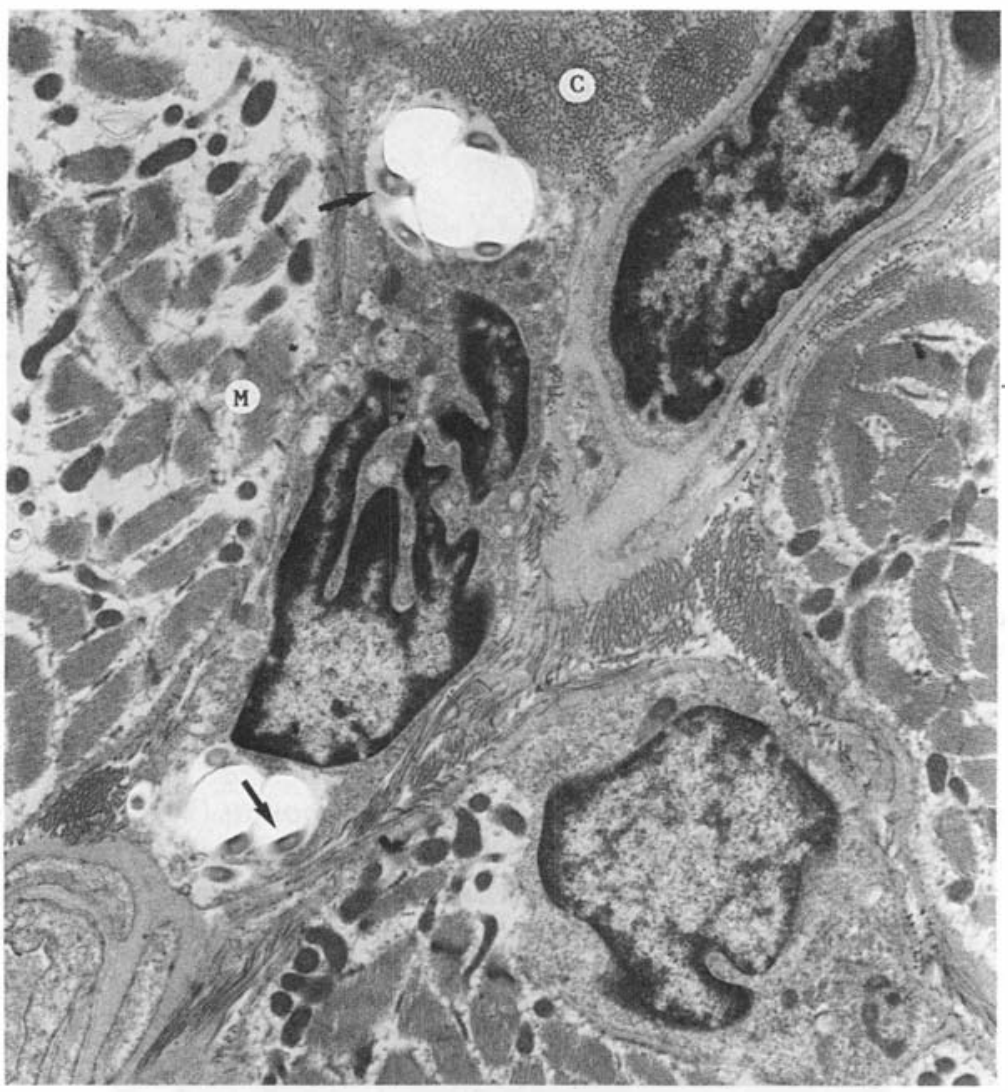

Figure 7. Within the cytoplasm of the infiltrating macrophage among the muscle cells (M), many M. leprae (arrows) could be seen. C, collagen. Electronmicrograph. $24000 \times$.

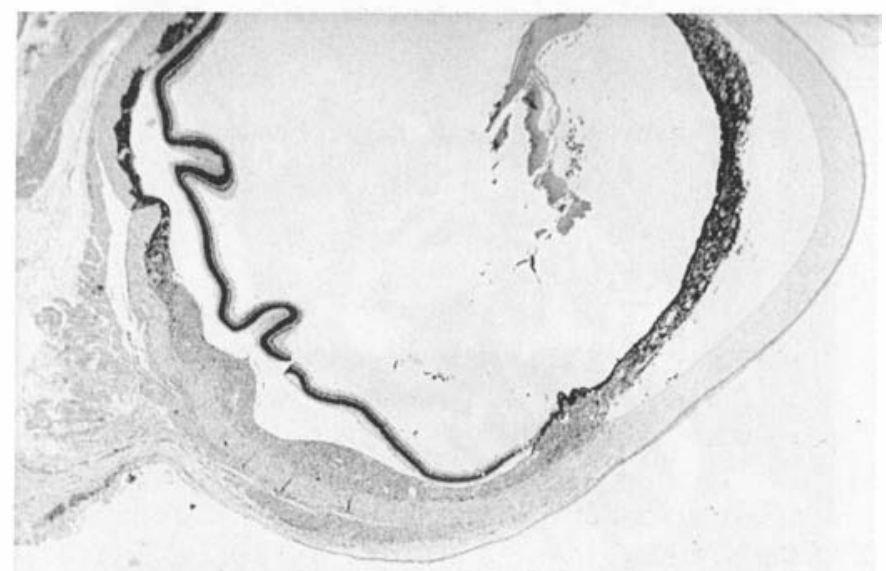

Figure 8. The cornea is not significantly infiltrated. But the iris, ciliary body, the choroid, even in the posterior segment of the eyeball, the sclera and the extraocular muscle are significantly infiltrated with inflammatory cells. HE $21 \times$. 


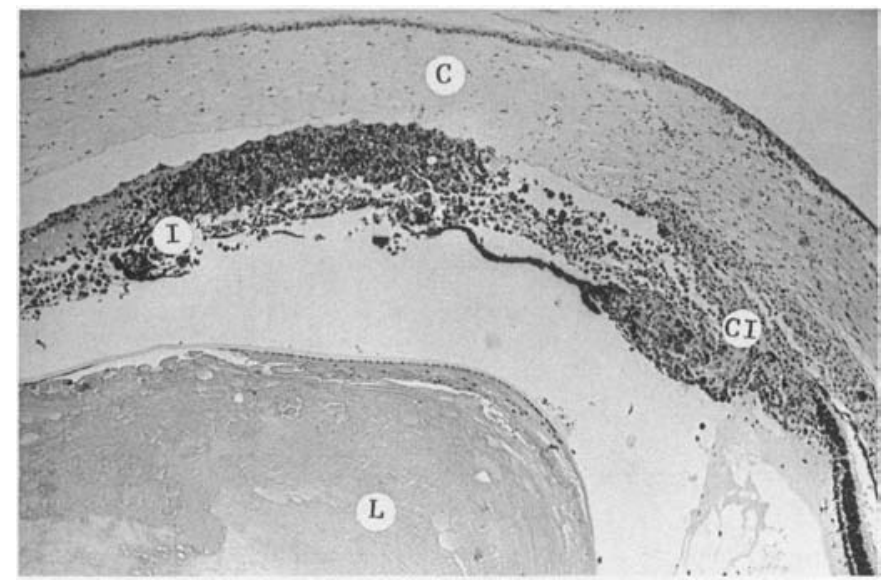

Figure 9. The cornea $(\mathrm{C})$ is significantly infiltrated with inflammatory cells only at the limbus region. The iris (I) and ciliary body (CI) are more or less destroyed and infiltrated by the inflammatory cells. Some fluid and cell exudates are filling the anterior chamber. The lens (L) shows Morgagni globules beneath the anterior capsule. HE $71 \times$.

endothelium, in the latter case sometimes in very large amounts (Figure 10). In one case, though the cornea of an eye in the +- lesion group was basically normal, several AFB were found in the superficial and basal epithelial cells of the cornea. Sometimes small round cells and macrophages had attached to the endothelium of the otherwise normallooking cornea (keratic precipitates, KP).

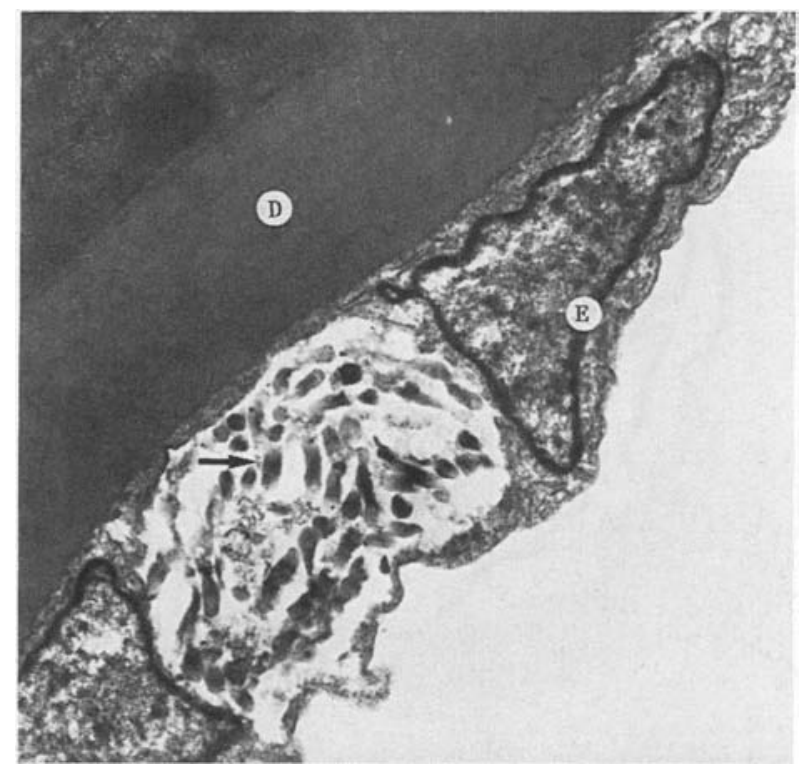

Figure 10. In the cytoplasm of this endothelium (E) swarms of $M$. leprae (arrow) indenting the nucleus are seen. D, Decement's membrane. EM $20000 \times$. 


\section{SCLERA}

In the sclera around the vicinity of ciliary bodies and choroid, there were large amounts of macrophages (Figure 11). There were also some scattered-cell infiltrations around small vessels and nerves within the sclera. In some cases the cell infiltrations in the choroid seemed to be continuous with the extraocular infiltrations, e.g. those in the conjunctiva and in the extraocular muscles through the sclera. AFB were found in the infiltrating cells and within the nerves traversing the sclera.

\section{TRABECULAR MESHWORK OF THE ANTERIOR CHAMBER ANGLE}

In the eyes with minimal alterations, the most significant change within the whole eyeball was almost always found around this region. Infiltration of small numbers of lymphocytes and plasma cells with or without plump macrophages were seen in the trabecular meshwork, around the Schlemm's canal and in the adjacent tissues of the ciliary body and iris (Figure 12).

When the infiltrations were denser, the cells dominating the picture were macrophages with abundant cytoplasm, within which there were different quantities of melanin granules. Sometimes the infiltrations tended to be continuous with the infiltrations in the limbus of the cornea and in the ciliary body and iris (Figure 13). These infiltrating cells were also found unattached in the anterior chamber. Among the free macrophages in the chamber, a giant cell was found in one case. AFB were present in the infiltrating cells.

\section{IRIS}

In some cases, the iris seemed normal, thin and without infiltrations. But in those with severe change, the iris was greatly thickened, infiltrated by large amounts of macrophage. The normal structure of the iris was obscured (Figure 9). In some cases the infiltrating cells were lymphocytes, plasma cells and various amounts of neutrophiles and eosinophiles.

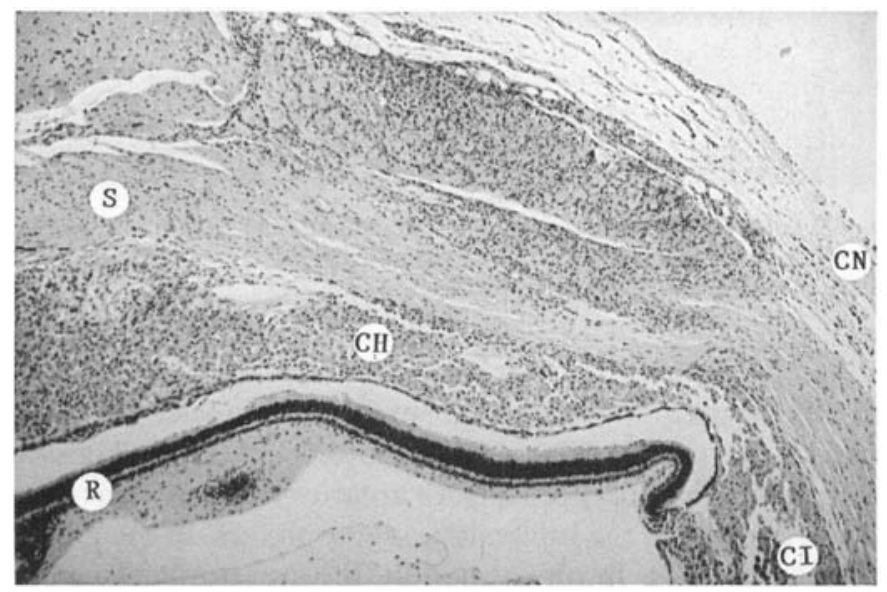

Figure 11. The sclera (S) adjacent to the heavy infiltrations of the choroid $(\mathrm{CH})$ and extraocular muscle $(\mathrm{M})$ is infiltrated with macrophages and other cells. $\mathrm{CN}$, conjunctiva, $\mathrm{CI}$, ciliary body; R, retina. HE $71 \times$. 


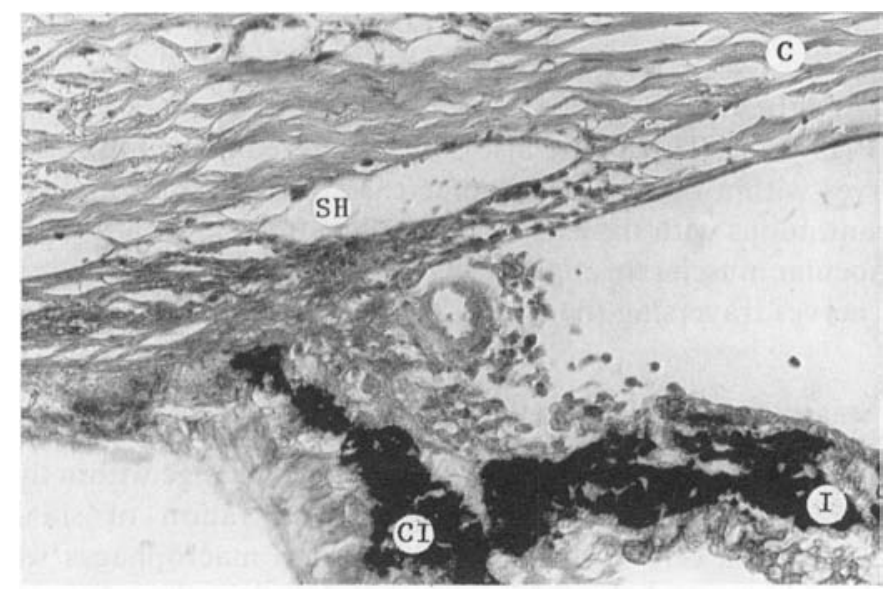

Figure 12. In the anterior angle area of this eye with slight lesions, tens of small macrophages and lymphocytes are seen in the meshwork, around the Schlemm's canal (SH), root of the iris (I) and in the adjacent ciliary body (CI). In the cytoplasm of these macrophages shown here, a large amount of AFB was seen. C, cornea. FF staining af ter depigmentation. $284 \times$.

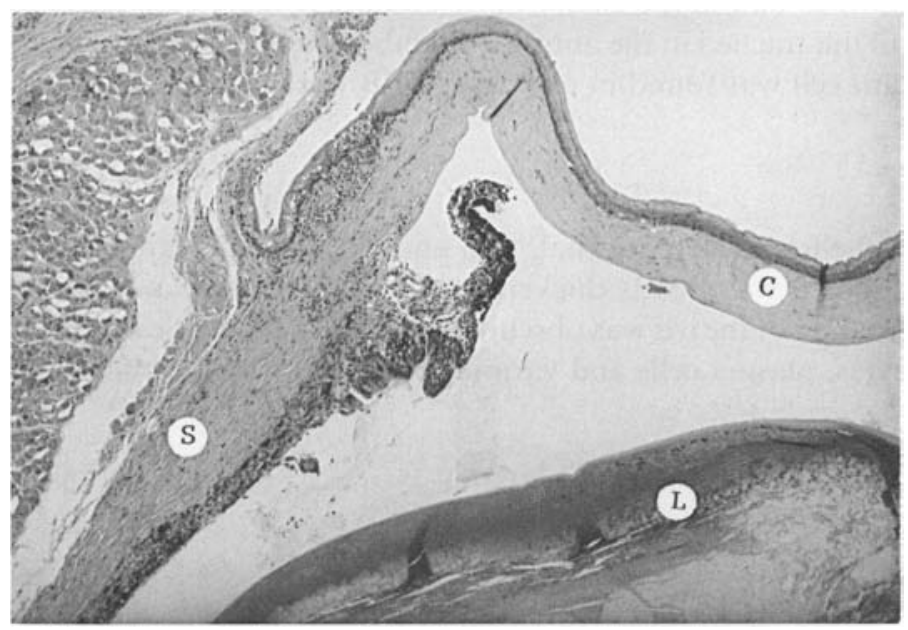

Figure 13. The anterior angle area is infiltrated with inflammatory cells. The infiltration extends to the limbus region of the cornea $(\mathrm{C})$ and seems to be continuous with that of the bulbar conjunctiva. The bulk of the cornea is not infiltrated. L, lens; S, sclera. Depigmented. HE $71 \times$.

Occasionally giant cells with one or several small or big nuclei were present among the other infiltrating cells (Figure 14). The sphincter muscle of the pupil of some of the iris was infiltrated with some inflammatory cells. Both anterior and posterior synechiae of the iris were seen. AFB were found in the infiltrating macrophages, in the giant cells mentioned above and in the muscle cells. In one armadillo the anterior surface of the iris and the anterior angle area were covered by endothelial cells continuous with those in the cornea (endothelialization). 


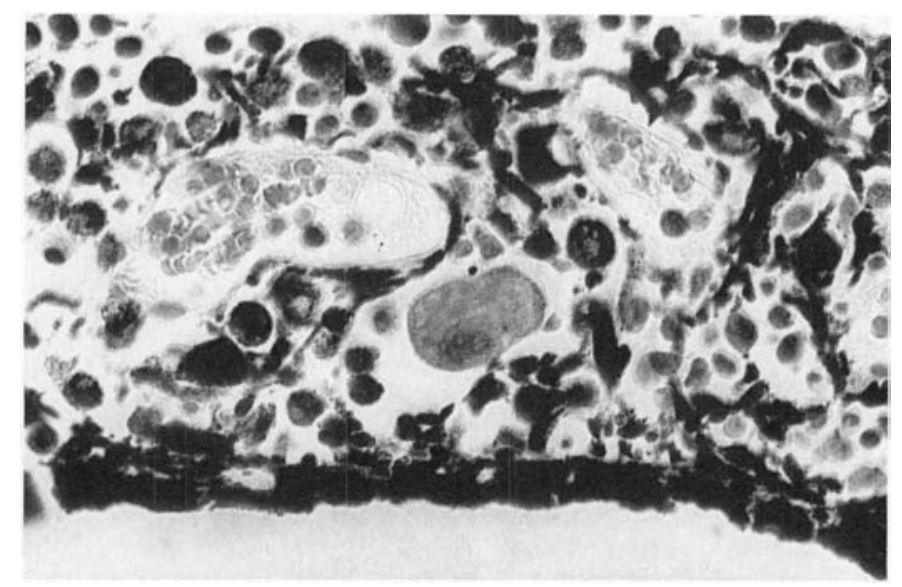

Figure 14. The iris is infiltrated with macrophages, lymphocytes and other cells. A giant cell with several nuclei is prominant among them. Within this giant cell, several AFB were seen. FF. $710 \times$.

\section{CILIARY BODY}

In some cases there were only small round cell infiltrations. But in others the whole ciliary body was swollen tremendously with large numbers of full-blown macrophages engulfing some melanin granules and with the tissue of the ciliary body destroyed. The muscular structure could not easily be recognized (Figure 9). Large amounts of AFB were found within these macrophages as well as in the pigment and non-pigment epithelial cells lining the ciliary processes (Figure 15). No fibrotic change was found.

\section{CHOROID}

In those cases with slight changes, the choroid was thin and without significant cell infiltrations. Only occasional, small round cells were found. In the cases with severe lesions, the choroid was thickened to several times the normal size, crowded with abundant macrophages and other cells, including some neutrophiles (Figure 16). The thickening appeared segmental and not uniform. Usually the anterior part was swollen the most, but sometimes only the posterior part was thickened and even more swollen. AFB were found in the infiltrating macrophages and stroma melanophores (Figure 17).

\section{RETINA}

In most cases the retina was observed to be devoid of a ny significant change. In armadillos the entire retina was abruptly joined to the epithelium of the ciliary body, and there was no obvious transitional zone corresponding to the ora serrata in humans. In seven eyes, in this anterior end of the retina adjacent to the heavily infiltrated choroid, many large macrophages had infiltrated. In another eye, at a place near the equator of the eyeball, where the choroid was heavily infiltrated with plump macrophages and the Bruch's membrane was destroyed, a lot of macrophages infiltrated the retina destroying the retina tissue (Figure 18). Large amounts of AFB were found there, both in the choroid and the 


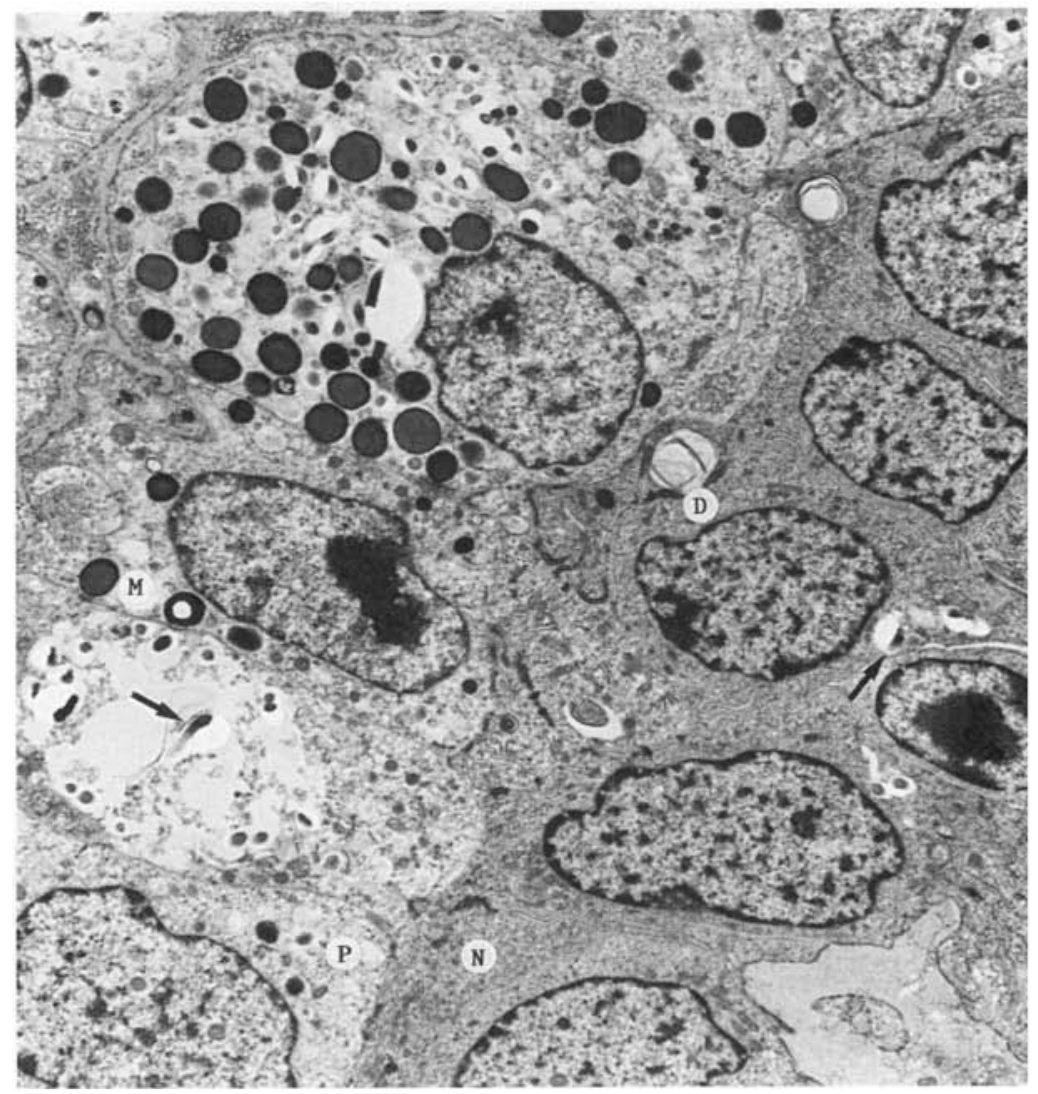

Figure 15. M. le prae (arrow head) in the cytoplasm of the pigmented (P) and non-pigmented (N) epithelium of the ciliary body. The melanosomes (M) and desmosomes (D) of the cells are very distinct. EM $12000 \times$.

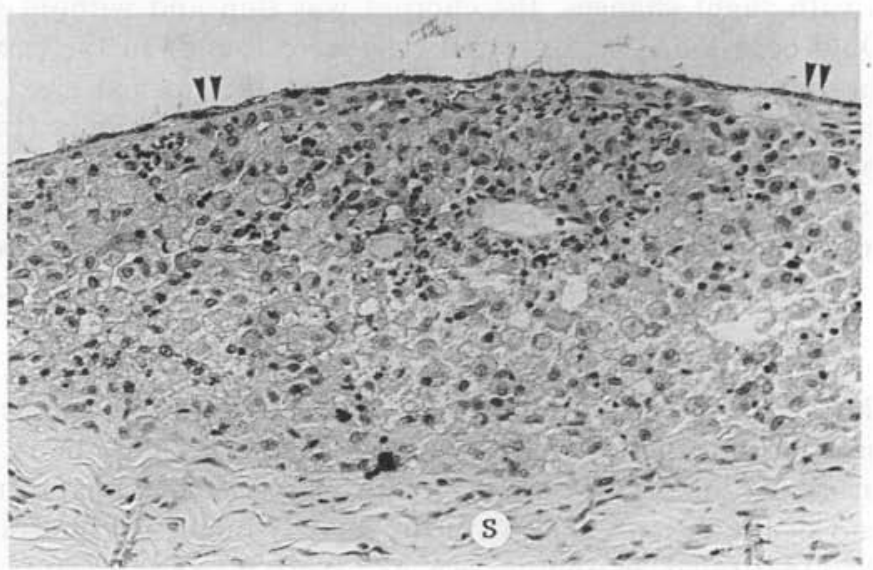

Figure 16. The choroid is thickened several times its normal size by the large gatherings of foamy macrophages. Within the cytoplasm large amounts of AFB were seen. Note the neutrophiles scattered among the macrophages. The pigment epithelium layer (arrows) is at the top and the sclera (S) is at the bottom of the picture. HE $284 \times$. 


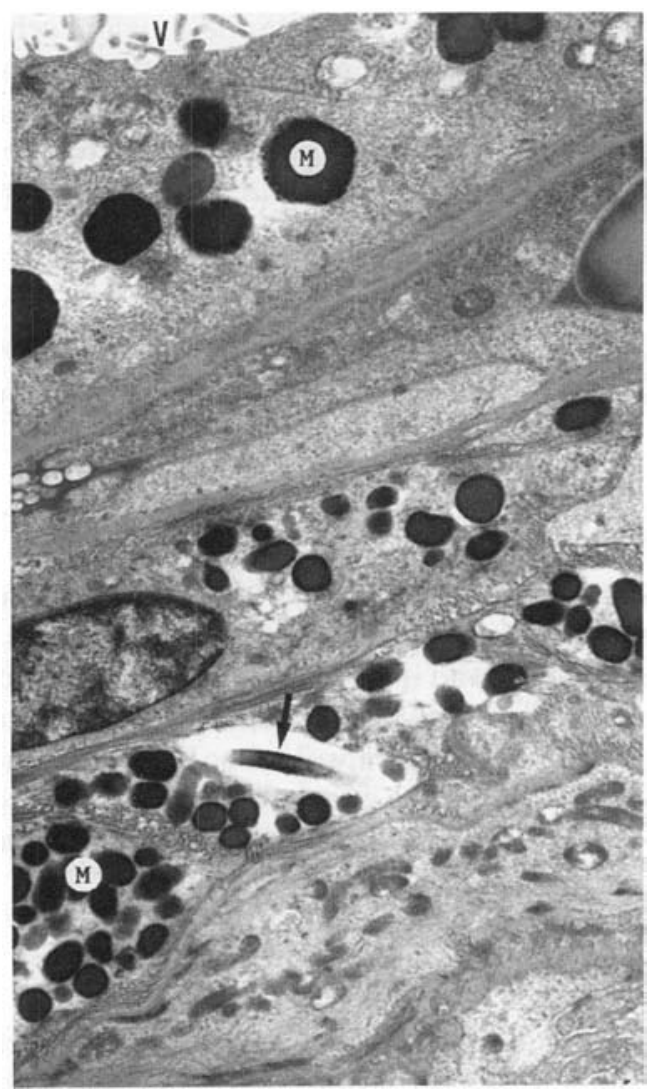

Figure 17. M. leprae (arrow) in the cytoplasm of a melanophore in the choroid. M, melanosome; V, microvilli of the pigment epithelium of the retina. EM $20000 \times$.

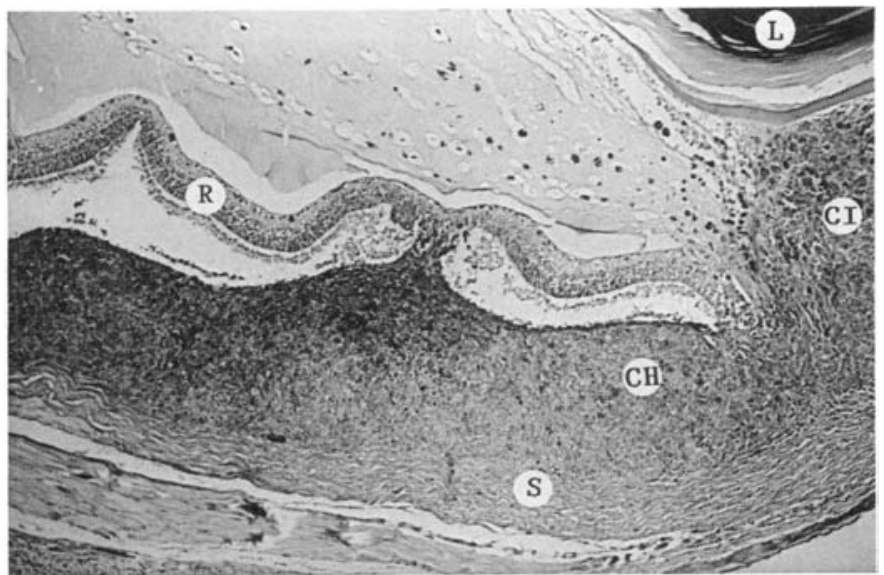

Figure 18. The retina $(\mathrm{R})$ here is adhered to the greatly thickened choroid $(\mathrm{CH})$, which is infiltrated with huge amount of foamy macrophages engulfing swarms of AFB. The lens (L) is at the top right-hand corner and the ciliary body $(\mathrm{CI})$ is also greatly engorged. S, sclera. FF. $71 \times$. 
retina. In nineteen cases $\mathrm{AFB}$ were also visible in the ganglion cell layer, in the inner and outer nuclear layer, between the photoreceptors and pigment epithelium of the otherwise normal retina (Figure 19). In several cases the retina was detached and fluid had gathered under the detached retina. In two cases segments of retina were atrophic; in those cases the retina had been reduced to a one-cell layer structure, but the adjacent choroid was normal.

\section{OPTIC NERVE}

No significant lesions were found in the optic nerves observed in 39 cases, but AFB were found in the meninges surrounding the optic nerve in five eyes and in the nerve itself in one (Figure 20).

\section{LENS}

Focal globular thickenings of the lens capsule with flattened epithelial cells were seen in thirteen lenses $(13 \%)$ of the 102 eyes with the lens in the sections. Morgagni globule or small vacuole formations were found in 34 cases. The thickening of the lens capsule and the degenerative change of the lens fibres in the same eye appeared in seven lenses. In four eyes a few AFB were observed under the capsule. One bacillus seemed to be located within a vacuole of the cell (Figure 21). In one eye the lens capsule was disrupted and the lens substance provoked an acute inflammatory reaction around it (phacogenic endophthalmitis).

\section{ENDOTHELIUM OF THE BLOOD VESSELS}

Some AFB were found in 18 eyes in the cytoplasms of the endothelial cells, or in the cells within the lumen of the blood vessels in various tissues, e.g. the limbus of cornea, choroid, sclera and iris.

In $85 \%$ of the cases, both eyes of an animal had the same degree of lesion. In $9 \%$ of the

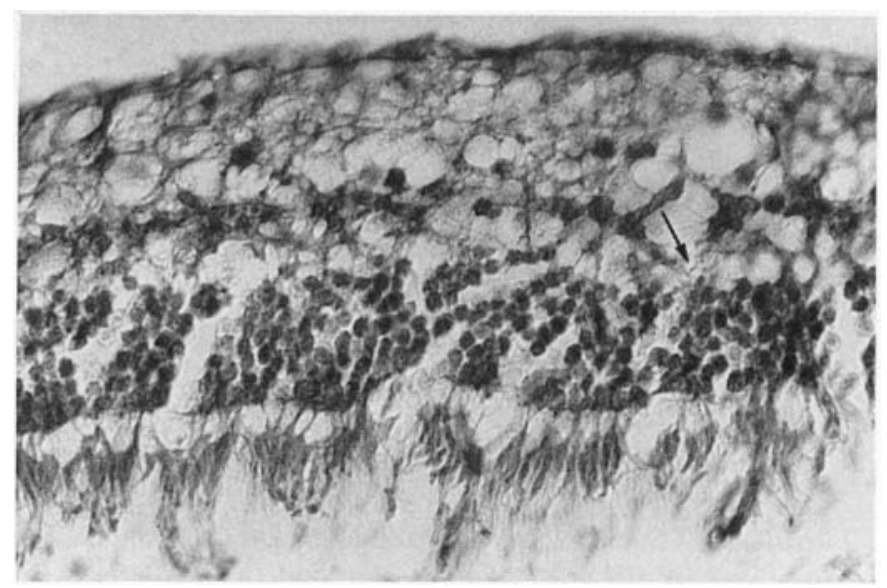

Figure 19. In various layers of the retina without significant lesions, $M$. leprae were observed, as shown here (arrow). FF. $710 \times$. 


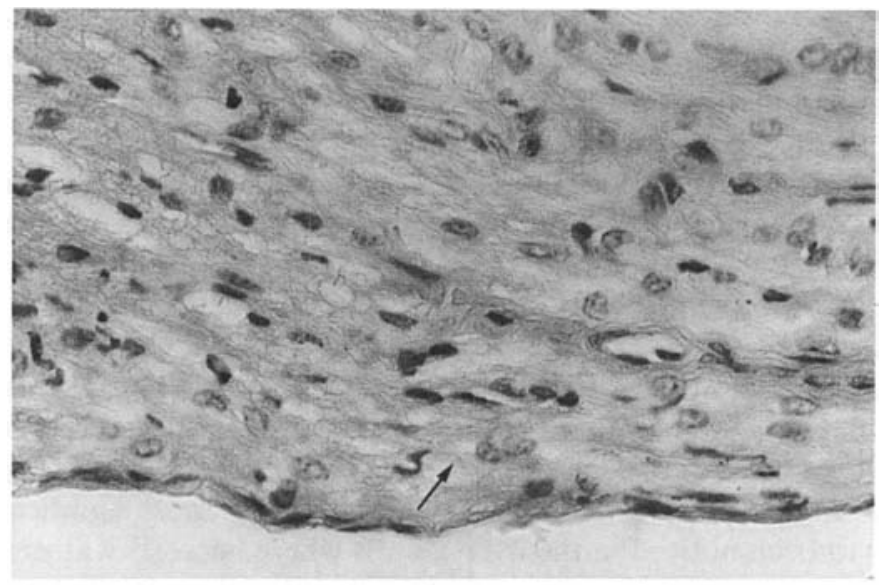

Figure 20. A M. leprae in the optic nerve. FF. $710 \times$.

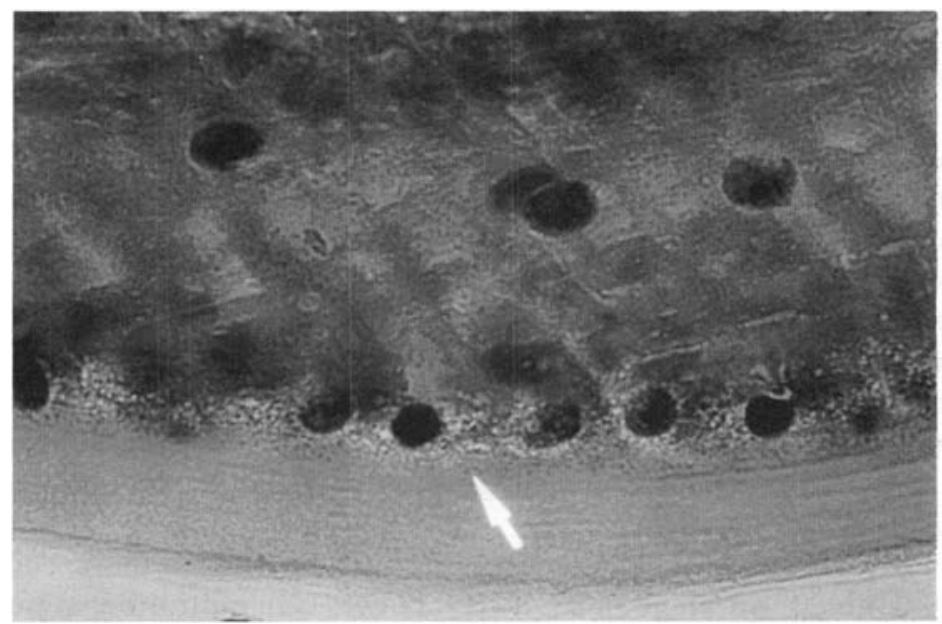

Figure 21. A M. leprae (arrow) in a vacuole of the epithelium of the lens under the capsule (C). FF. $710 \times$.

animals the degrees of the lesions of their two eyes were not identical, but of an adjacent degree. In only $6 \%$ out of all the armadillos were the eye lesions differing by two or more degrees.

\section{Discussion}

Of the 66 armadillos which were experimentally infected with $M$. leprae, no AFB were found in the liver or in the spleen in five animals at the time of their deaths. However, in the eyes of these five animals, minimal cell infiltrations around the anterior angle area 
were found (group +- ), although no AFB were found in these eyes. Since AFB were observed in other eyes in the +- group, the +- lesion may represent either an early and slight leprosy inflammation or a mild, nonspecific reaction to some other irritants.

All the other 61 experimentally infected armadillos in this study had disseminated leprosy infections upon death. The amount of the bacilli in their spleens and liver and the presence of granulomata in the soft tissue within their bodies seen in post-mortem examinations verified the nature of their systemic lepromatous infection. The eye lesions are simply a part of the manifestations of the general infection.

The granulomatous reaction in the intraocular and extraocular tissues was manifested by macrophage infiltrations, and the cytoplasm of these cells was sometimes not extreme, and at other times very abundant and foamy. Among these cells there were usually some neutrophiles. Often there were some lymphocytes and plasma cells intermingled, whether the lesion was in the eyelid, in the iris, in the choroid, or most significantly in the iris. Neutrophiles had been noticed in the liver lesions where necrosis was present ${ }^{6,7}$ and had been mentioned as a particular component of cell infiltrations in the eye. ${ }^{1}$ In the eyes of this study the neutrophiles were present both in the intraocular and the extraocular lesions where there were no obvious signs of necrosis. What this exudation means remains to be determined. There were two possible routes for the bacilli to get into the anterior angle area, namely invasion through the cornea or adjacent extrabulbar tissues (via nerves or by direct extensions from conjunctiva, extraocular muscles and fibra-fatty tissues) and haematogenous seedling.

When the lesions within the eye were slight (group +- ), they were almost always confined to the anterior angle region, with cell infiltrations around the trabeculae, at the limbus region of the cornea and in the adjacent ciliary body and the root of the iris. AFB were also found in these places, sometimes in quite large numbers, even when the cell infiltration was not very severe.

When the lesions became more severe the ciliary body and iris were affected more. The lesions in the choroid, when present, seemed to be commonly more severe in the anterior part, though in some cases the posterior part was equally, if not more severely, affected. The lesions in the anterior angle area might be the initial intraocular lesions in these intravenously infected animals.

Inflammatory cells were seen only occasionally in the stroma in the corneas of 30 of the 127 eyes examined. Although in 89 of the 127 eyes cell infiltrations were found in the limbal regions, the cell infiltrations were not dense and there was no frank granuloma or significant lepra cell infiltrations in the stroma of the cornea in the central part, as seen by others. ${ }^{8-11}$ In comparison to the severity of the lesions with that in other eye tissues, the corneal lesions were slight. Although AFB were found in the epithelium of the cornea, it seemed that $M$. leprae cannot have entered the eye initially through the cornea.

In view of the fact that the lesions of the extraocular tissues in the vicinity were insignificant in those eyes with slight leprosy lesions in the angle area, the continuous invasion route did not seem to be of significant importance.

The large amount of AFB within the endothelia of the cornea and epithelia of the ciliary body in some cases was of interest. That meant that the bacilli could multiply and accumulate within them and shed into the fluid phase within the eyeball.

The intraocular circulation of the aqueous humorwith the trapping and concentrating of particulate matters and cells (might be bacilli-laden) between the meshworks of trabeculae might be a factor in the early cell infiltrations there; the lower temperature of 
the anterior parts of the eyeball might also be a factor in the early lesions there. The ciliary body receives an enormous blood supply. Bacilli in the bloodstream might gather more easily at the site where the blood supply is greater. Choroid also receives a large amount of blood; nearly as much as that of the ciliary body. If only the number of AFB in the blood supplied to these parts were concerned, as the ultrastructures and permeability of the capillaries of choroid and ciliary body are about the same, the severity of the lesions in these two parts should be similar. That was true in some cases we examined, but we observed cases in which the lesions in these two structures were not the same, usually with heavier lesions in the ciliary body, but also with heavier lesions in the other. The cause of these differences might be solely a matter of chance, but tissue preference or other factors must also be considered.

Retinal lesions in leprosy have rarely been seen. ${ }^{9-11}$ In the present studies, retinal lesions were observed in 8 of 125 cases. In seven cases, the lesions were at the beginning part of the retina adjacent to the ciliary body and choroid where there were heavy macrophage infiltrations. In another animal, a focal lesion was found in the retina at about the equator of the globe, where there were also heavy macrophage infiltrations in the choroid. Here the retina and the Bruch's membrane were destroyed. The retinal lesions in these 8 cases were obviously extensions of the adjacent choroid and/or ciliary body lesions. But AFB were found, too, in the retina without obvious lesions in another 19 cases. These AFB might be blood borne, since bacteremia might be a constant event in this systemic infection. The reason why so few retinal lesions were seen, after the possible constant bacteremia, might be attributed to the properties of the blood-retinal barrier or to some other properties of the microenvironment in the retina.

The finding of a few AFB within the lens in four eyes was unexpected. It is not easy to imagine how the bacilli could penetrate the thick lens capsule. Since they were seen under the microscope at the levels of the cell organelles and some were in vacuole-like structures and not at the top of tissue, they probably were not dislodged bacilli resettled there during slide preparations.

The endothelialization of the anterior surface of iris in one case, the phacogenic endophthalmitis in one and the focal atrophy of the retina in two cases might not be directly related to leprosy infection. The sarcocystis seen in the muscle fibres in several armadillos is a common parasite of various laboratory animals and sometimes of human beings, and it usually does not provoke strong reactions, if any, in the host.

Applying the findings of the eye lesions of the armadillos to human patient treatment may not be done directly. But since we saw small amounts of cell infiltrations around the anterior chamber angle, and some free cells in the chamber with acid-fast bacilli in them, and as the early lesions in the intraocular tissues showed no obvious inflammatory reactions in the iris in the armadillos, we may suggest that the early lesions in human eyes could also be similar to those seen in the armadillos. Other findings in the eyes of armadillos infected with $M$. leprae are also similar to the eye complications in leprous patients. As in humans, the ocular involvement is mostly symmetrical, just as it happens that $94 \%$ of the armadillos showed similar grades of ocular involvement in both eyes. ${ }^{12}$ The inflammation of the $M$. orbicularis has been shown by electromyography ${ }^{13}$ and is one of the reasons for lagophthalmus. And if the lacrimal gland in humans were involved in the disease as in the animal, it must be expected that tears are infectious, especially in multibacillary patients. We cannot say whether the inflammatous infiltrations of the extraocular muscles may cause paralysis in armadillos, but in humans such complications have not been observed. 
The early, distinct involvement of the anterior segment, of the trabecule and of the ciliary body in the armadillo eye is very similar to the situation in the human eye. This is also true for the presence of AFB in the aqueous humor. ${ }^{14}$ Keratitic precipitates and iritis are common in leprous patients too. ${ }^{15}$ The way in which the AFB enter the eyeball has often been discussed, but we would like to emphasize that the AFB may also leave the eye via nerves or vessels, as shown in the armadillos. Scleritis is also a common eye complication in leprosy in humans. However, in the lens of inactive leprous patients no AFB were found. ${ }^{16}$ To the best of our knowledge the lens of active multibacillary cases have not been examined with that in mind. Similar to leprosy in humans, ${ }^{17,18}$ the involvement of the posterior segment has been observed in only a few cases.

Our findings, according to which the grade of the lesion in the eye of the armadillo was correlated with the number of AFB found in it, may not easily be transferred to ocular leprosy among humans. Therefore we think that routine studies of eye tissues taken from patients during surgery, like iridectomies, cataracts, enucleations and other operations including autopsies, would be much more useful than the study of eyes of an animal in the prevention of blindness and for the elucidation of ocular problems in leprosy patients.

\section{Acknowledgments}

This study was supported financially by the German Leprosy Relief Association (DAHW) and assisted, in part, technically by Amauer Hansen Institute, Würzburg, Federal Republic of Germany. Lepra are also grateful to the German Leprosy Relief Association (DAHW) for financial assistance in producing the illustrations.

\section{References}

1 Courtright P, Johnson GJ (eds). Prevention of blindness in leprosy. London: The International Centre for Eye Health, 1988; 25-8.

2 Kirchheimer WF, Storrs EE. Attempts to establish the armadillo (Dasypus novemcinctus Linn.) as a model for the study of leprosy. Int J Lepr, 1971; 39: 693-702.

${ }^{3}$ Storrs EE. The nine-banded armadillo: a model for leprosy and other biomedical research. Int J Lepr, 1971; 39: $703-714$.

4 Convit J, Aranzazu N, Pinardi ME. Leprosy in the armadillo clinical and pathological aspects. The armadillo as an experimental model in biomedical research. Washington D.C.: Pan American Health Organization, 1978; 41-8.

5 Jones RL, Ryan TJ. Demonstration of leprosy bacilli in the eyes of experimentally infected armadillos: a comparison of five melanin bleaching methods. Med Lab Sci, 1986; 43: 211-14.

6 Job CK, Kirchheimer WF, Sanchez RM. Liver lesions in experimental lepromatoid leprosy of the armadillo. A histopathologic study. Int J Lepr, 1978; 46: 1-8.

7 Job CK, Sanchez RM, Hastings RC. Manifestations of experimental leprosy in the armadillo. Am J Trop Med Hyg, 1985; 34: 151-61.

8 McDougall AC, Rees RJW. 1. Ocular histopathology in animals experimentally infected with Myobacterium leprae and M. leprae-murium. 2. Mycobacterium leprae infections in the 9-banded armadillo (Dasypus novemcinctus L.). Br J Ophthal, 1978; 62: 516-24.

9 Jakob M. 'Ocular leprosy in the armadillo and man-a histopathological study'. Abstracts of the 13th International Leprosy Congress 88 Bologna: Organizzazione per la Cooperazione Sanitaria Internationale 1988; 263.

10 Malaty R, Tongni B. Corneal changes in nine-banded armadillos with leprosy. Invest Ophthal Vis Sci, 1988; 29: 140-5.

11 Malaty R, Walsh GP, Meyers WM, Binford CH, Job CK. Ocular leprosy in nine-banded armadillos. Invest Ophthal Vis Sci, 1986; 27: 38. 
12 Brandt F, Adiga RB, Pradhan H. Lagophthalmus und hintere Synechien der Iris bei der Behandlung der Lepra mit Diamino-diphenyl-sulfon. Klin Mbl Augenheilk, 1984; 184: 28-31.

13 Slem G. Electromyographic investigation in patients with leprosy. Ankara Univ Tip Fak Göz Klin Yill, 1970; 22: $120-32$.

14 Agrawal LP. Aqueous humor examination in granulomatous iridocyclitis. Docum ophthal, 1960; 14: 97-110.

15 Brandt F, Zhou HM, Shi ZR, Rai N, Thuladar L, Pradhan H. Histological findings in the iris of DDS-treated leprosy patients. Br J Ophthal, 1990; 74: 14-18.

16 Brandt F, Kampik A, Malla OK, Pokharel RP, Wos J. Blindness from cataract formation in leprosy. Dev Ophthal, 1983; 7: 1-12.

17 Choyce DP. Diagnosis and management of ocular leprosy. Br J Ophthal, 1969, 53: 217-223.

18 Somerset EJ, Sen NR. Leprosy lesions in the fundus oculi. Br J Ophthal, 1956; 40: 167-71. 